\title{
sciendo
}

\section{The Effect of Eight Weeks of Sling-Based Training with Rotational Core Exercises on Ball Velocity in Female Team Handball Players}

\author{
by \\ Kenneth Stakset Dahl ${ }^{1}$, Roland van den Tillaar ${ }^{1}$
}

\begin{abstract}
The purpose of this study was to investigate whether sling-based training focused on rotational exercises would improve shooting performance in outfield handball players during the competitive season, and whether changes in performance were related to altered levels of core strength and rotational velocity. Twenty-five female outfield handball players (mean age $19.5 \pm 2.0$ years, height $1.72 \pm 0.06 \mathrm{~m}$, body mass $71.5 \pm 8.6 \mathrm{~kg}$, training experience $10.3 \pm 2.4$ years), performed $7 \mathrm{~m}$ shots, with and without a run-up, and jump shots. Maximal ball velocity, peak rotational velocity of the trunk with different loads and 1RM in a core strength test were measured before and after an 8-week training intervention. Players were divided into a sling-based and a plyometric/sprint training (control) group that trained three times per week for 8 weeks. The main findings were that sling-based training increased ball velocity by on average 3.2\% across three techniques tested, while shooting performance decreased by $3 \%$ in the control group. However, both training groups demonstrated increased peak rotational velocity with different loads, but not the calculated 1RM core strength after the training period. It was concluded that sling-based training with rotational core exercises could improve maximal ball velocity in female handball players during a competitive season by around $3 \%$. However, this increased ball velocity may have been caused by increased angular velocity in the core, rather than absolute maximal core strength. It is suggested that sling-based training has impacted timing variables of the different involved segments, or possibly power transfer between segments, which may explain the enhancement in ball velocity.
\end{abstract}

Key words: strength, performance, conditioning, stability, outfield players.

\section{Introduction}

Shooting is an ability that is utilized in many sports, such as baseball, basketball, and team handball. In team handball, different shooting techniques are applied, including jump shots and standing shots, with or without a runup, which are considered fundamental skills for handball players (Wagner et al., 2011). These techniques are mainly used to score goals and are a large part of the repertoire of skilled players (Wagner et al., 2010). Shooting is classified as a fast-discrete complex movement, involving several joints and muscles, and therefore the player must deal with numerous degrees of freedom during a shooting motion with a distinct beginning and end (van den Tillaar and Ettema,
2007). This means that several joints and muscles must be coordinated into one coherent action to maximize shooting performance.

The coherent action in shooting can be explained through the proximal-to-distal movement in various shooting techniques, demonstrated by both Wagner et al. (2011), and van den Tillaar and Ettema (2009b). These studies suggest that variables in core movements will impact the sequence, and thereby, shooting performance (van den Tillaar and Ettema, 2007; Wagner et al., 2011), as the core consisting of the trunk and pelvis area acts as a bridge between the upper and lower extremities (Willardson, 2007). Wagner et al. (2011) also discovered a high correlation between both maximal pelvic and

1 - Department of Sport Sciences and Physical Education, Nord University, Levanger, Norway. 
trunk angular velocity, as well as moderate correlation of timing of the maximal internal trunk rotational angle, with maximal ball velocity. Jöris et al. (1985) theorized that the acceleration of a proximal segment (e.g. trunk) through the concentric contraction of involved muscles would make muscles in the succeeding, distal segment (e.g. shoulder) rapidly contract eccentrically, leaving them pre-stretched. When these muscles in turn contract concentrically, due to their prestretched characteristics, they do so with higher power output. Then, through a chain of segmental movements, the momentum of enhanced power output can be transmitted from segment to segment, eventually resulting in greater ball velocity. Therefore, it seems beneficial to train core muscles regularly, as strong muscles in the core logically would act as an important foundation for transferring joint movements of the lower extremity to the upper extremity, thereby achieving the highest ball velocity possible.

However, a few studies have examined whether core training can increase sports performance (shooting, throwing, swimming and running), and so far, these have had varying success (Goulet and Rogowski, 2018; Manchado et al., 2017; Scibek, 1999; Stanton et al., 2004). Both Scibek (1999) and Stanton et al. (2004) reported increased levels of core strength in athletes, but neither study found increased performance in swimming or running, respectively. The lack of progress in these studies could be due to the level of the specificity of the chosen exercises, loading, and the intensity applied. However, another explanation might be that core muscles do not play the same part in repetitive, long-lasting activities, such as running and swimming, as they would in short, explosive movements like shooting, throwing and kicking.

On the other hand, other studies (Genevois et al., 2014; Saeterbakken et al., 2011; Seiler et al., 2006; Stray-Pedersen et al., 2006) that investigated the effect of core training on fastdiscrete complex movements (shooting, serving, kicking and maximal golf club-head swing velocity), all observed improvements ranging from 2 to $4.9 \%$. The core strength programs were different between studies; Manchado et al. (2017) carried out floor-based core training, whereas the other studies used sling-based training (Goulet and Rogowski, 2018; Saeterbakken et al., 2011; Seiler et al., 2006; Stray-Pedersen et al., 2006). Apart from the differences in the applied training methods, the studies all trained both stabilizing and rotational exercises, therefore none of the studies could explain which types of exercises (and to what extent) contributed to the enhanced performance. In addition, none of them could explain if the increased performance was due to enhanced core strength, since no strength test was included for the core.

Therefore, the main aim of this study was to investigate whether sling-based training focusing on rotational exercises would improve shooting performance in outfield handball players during the competitive season. The second aim of this study was to investigate whether the results were related to altered levels of core strength and peak rotational velocity.

\section{Methods}

To investigate the effect of sling-based training on shooting velocity, a pre-test post-test randomized-groups design, with a training intervention between tests, was carried out by both an experimental and a control group. Both groups carried out a training program lasting 8 weeks, either sling-based training or plyometrics/sprints, so that the chosen form of the exercise modality would act as the independent variable, eliminating the possibility that additional exercise/no additional exercise would impact the results.

\section{Participants}

Twenty-five female outfield handball players (mean age $19.5 \pm 2.0$ years, body height $1.72 \pm 0.06 \mathrm{~m}$, body mass $71.5 \pm 8.6 \mathrm{~kg}$, training experience $10.3 \pm 2.4$ years) playing in the first and the second division of the Norwegian national league volunteered to participate in this study. Participants were fully informed about the complete test protocol; informed written consent was obtained from participants or their parents when they were under the age of 18 , prior to the pre-test. The study was approved by the Norwegian Centre for Research Data (NSD) and conducted in accordance with the current ethical standards for sports and exercise research.

\section{Test Procedures}

Participants began the test by carrying out their regular 10-min warm-up, which included 
running, jumping, and shooting, followed by a shooting test involving a $7 \mathrm{~m}$ penalty shot, a $7 \mathrm{~m}$ shot with a two-step run-up, and a jump shot (also with a two-step run-up) at $8 \mathrm{~m}$; these were always completed in this order. Maximal ball velocity was measured by a Doppler radar gun (Stalker ATS II, Applied Concepts Inc., Plano, TX). The radar gun was located $11 \mathrm{~m}$ away from the target participants were aiming at, placing the radar gun in a straight line between the target, the thrower, and the gun. It measured speed with accuracy of $0.028 \mathrm{~m} / \mathrm{s}$ within a $10^{\circ}$ field.

Participants were instructed to throw as hard as possible and to try to hit a 0.5 by $0.5 \mathrm{~m}$ square target at a height of $1.65 \mathrm{~m}$ (van den Tillaar and Ettema, 2003). Participants used a regular senior handball (mass: $0.36 \mathrm{~kg}$, circumference: 0.54 $\mathrm{m})$ for all attempts. All participants performed one type of a shot until told otherwise, not knowing the total number of successful attempts needed to complete the test. Three successful attempts (hitting the target) per shooting technique were considered enough, unless their performance gradually increased between each successful attempt; if that were the case, they would keep on shooting until their performance levelled out. By doing so, it was possible to control for a learning/adjustment effect that could influence the initial performance. The three best performances were picked, to make sure that the best performance from the pre-test was used, so that possible changes on the post-test would reflect the influence of training as correctly as possible. Between trials, participants had approximately $1 \mathrm{~min}$ of rest to avoid the interference of fatigue. After 8 weeks of training, participants performed a post-test at approximately the same time of day, and on the same day of the week, to avoid scheduled training or matches that could affect the recordings differently between tests.

After the shooting test was completed, participants carried out a core strength test, emphasizing rotational peak velocity around the longitudinal axis, with a resistance of 5, 10, 15, and $20 \mathrm{~kg}$ on both sides. All participants sat on a bench (without support) $0.75 \mathrm{~m}$ from the apparatus, with a band chained to the applied resistance over one shoulder at a time, holding the band with the opposite hand across their upper body. Participants were instructed to rotate the trunk as fast as possible around the longitudinal axis to mimic the shooting movement with the trunk and slowly return back to the starting position. They always had to hold their feet above the ground during testing, so they could not use them to generate power from the ground (Figure 1). For every load, participants performed three attempts each, with a 1 min rest interval between each load.

Peak rotational velocity was recorded with a linear encoder (ET-Enc-02, Ergotest Technology AS, Langesund, Norway) attached to the weights and calculated using software Musclelab 10.5.57.4354 (Ergotest Technology AS, Langesund, Norway) with reliability of 0.94 (ICC 3,1 ) established over the three repetitions for all weights at the pre-test. Based on the data collected on this test with the four different loads, a loadvelocity relationship was established for each participant, for both the pre- and post-test. The mean peak velocities based on three attempts were calculated, and then used for further analysis. A load-velocity relationship was then established as a product of the load, and the rotational velocity at that load. Based on the athlete's performance for the various loads, a linear regression was used to calculate the theoretical 1RM for each participant. The $x$ variable was set as $0.2 \mathrm{~m} / \mathrm{s}$, which indicates the velocity at which $1 \mathrm{RM}$ is theoretically attainable (based on pilot data). To calculate 1RM, the following formula was used:

$$
y=a * 0.2 \mathrm{~m} / \mathrm{s}+b
$$

Both the coefficient of $x$ (a) and the $y$ intercept (b) are individual values for each participant. To establish $\mathrm{a}$ and $\mathrm{b}$ for each participant in the linear equation, a scatter plot with an added regression line in Excel was used. Then, when replacing $x$ with $0.2 \mathrm{~m} / \mathrm{s}$, the formula for $1 \mathrm{RM}$ was complete, and the load-velocity relationship for maximal performance was established for each participant.

\section{Training procedures}

After testing was completed, participants were matched according to their mean performance for shooting with a run-up and then randomly assigned to either the sling-based training group $(n=13)$ or the control group $(n=$ 12). The sling-based group used adjustable Redcord Mini slings (Redcord AS, Kilsund, Norway, www.redcord.com), as well as $1.5-\mathrm{cm}$ - 
wide Abilica elastic bands (Mylna Sport AS, Mjøndal, Norway, www.abilica.no) in their training, whereas the control group used no extra equipment.

The sling-based group carried out a twophased training protocol, consisting of four exercises at every stage, where the second phase was based on the same exercises from phase one, but with an altered movement pattern or with added resistance (Figure 2). Participants were encouraged to increase the velocity gradually for each repetition and session, while their technical performance of the exercises progressed. This is in accordance with the principles of loading, where they gradually increased the performance intensity while they developed motor control in these exercises, until they could complete the whole exercise with maximal effort.

The training period lasted 8 weeks from January to March, which was in the second part of the competitive season. In the first 5 weeks, the slingbased training group performed three sets of 10 repetitions per exercise on each side, followed by 3 weeks with four sets of 4-6 repetitions on both sides with maximal effort, to further work on their explosive performance. An instructor was present during every session to guide participants, regarding both their execution, as well as how to make the exercises within each phase gradually more difficult. Participants were also told to maintain a stable core through the activation of both the core and gluteus muscles, so that they could make the desired rotation around the spine, leaving the possibility of back and hip flexion and extension as small as possible, and only when it was intentional.

The control groups completed a plyometric/sprints program for the lower body based on the studies of Marques et al. (2013), and van den Tillaar et al. (2015). Before the training period began, participants in both groups received instruction for how to carry out the applied exercises, emphasizing correct execution, so that they were familiar with their respective training protocol. The control group carried out a plyometric/sprints program (Table 1), emphasizing variations of one and two-legged jumps and sprinting, for 8 weeks. According to van den Tillaar et al. (2015), plyometric/sprint training for the lower body should not affect maximal ball velocity, which was the main reason for choosing this exercise modality, while this group still accumulated the same amount of training as the sling-based training group. This ensures that potential improvements in shooting performance were not due to an increased amount of training, and since both groups trained an equal amount, it should be easier to establish whether sling training had any effect on performance.

\section{Statistical analysis}

The Kolmogorov-Smirnov test was used to determine any obvious effects and estimate the distribution of the data. Homogeneity of variance was tested using the Levene's test. A mixed model (factorial) analysis of variance (ANOVA) with repeated measures was conducted (2: sling-based plyometric/sprint training group $* 2$ : pre- and post-test) to investigate the impact of the training modality on shooting performance of the three different shots, the peak rotational velocity at each load in the core strength test and the calculated 1RM. In addition, Pearson correlation coefficients were calculated to investigate whether the change in shooting performance was related to changes in core strength. The test-retest reliability (three repeats per condition measured during the pretest), as indicated by intra-class correlations (ICC), was $\geq 0.95$ for ball shooting velocity with the different shots and $\geq 0.86$ for the core strength tests with different loads. The effect size used and reported in this study was partial eta squared $\left(\eta^{2}\right)$, where $0.01 \leq \eta^{2}<0.06$ constituted a small effect, $0.06 \leq \eta^{2}<0.14$ constituted a medium effect, and $\eta^{2}$ $<0.14$ constituted a large effect (Cohen, 1988). The data were analyzed in SPSS Statistics 23 for Windows (SPSS Inc., Chicago, IL, USA), with the alpha $(\alpha)$ for all statistical tests set at $p \leq 0.05$ to determine statistical significance.

\section{Results}

Shooting performance during the pre-test was, on average for the whole group, $19.9 \pm 1.5$, $21.7 \pm 1.3$ and $20.6 \pm 1.1 \mathrm{~m} / \mathrm{s}$ for standing shots, shots with a run-up and jump shots, respectively. Unfortunately, five players (two in the plyometric/sprint and three in the sling-based training groups) had to withdraw from the study, either because of an injury/illness that developed right after the pre-test, or due to lower leg strain injuries developed in the plyometric/sprint training group (control). 
Table 1

Plyometric/sprint training program for eight weeks with two training sessions per week.

\begin{tabular}{|c|c|c|c|c|c|c|c|c|}
\hline \multirow[b]{2}{*}{ Exercise } & \multicolumn{8}{|c|}{ Training session } \\
\hline & 1 & 2 & 3 & 4 & 5 & 6 & 7 & 8 \\
\hline $\begin{array}{l}2 \text { legged jumps (without bending the } \\
\text { knees) }\end{array}$ & $3 \times 20$ & $3 \times 20$ & $3 \times 20$ & $3 \times 25$ & $3 \times 25$ & $3 \times 25$ & $3 \times 30$ & $3 \times 30$ \\
\hline $\begin{array}{l}2 \text { legged jumps (while bending the } \\
\text { knees) }\end{array}$ & $3 \times 10$ & $3 \times 10$ & $3 \times 10$ & $3 \times 10$ & $4 \times 10$ & $4 \times 10$ & & \\
\hline $\begin{array}{l}2 \text { legged jumps as far as possible (while } \\
\text { bending the knees) }\end{array}$ & & & & & & & $3 \times 10$ & $3 \times 10$ \\
\hline Hop on one leg, short and quickly & $3 \times 10$ & $3 \times 10$ & $3 \times 10$ & $3 \times 10$ & $2 \times 10$ & $2 \times 10$ & $3 \times 10$ & $3 \times 10$ \\
\hline 1-legged jumps as high as possible & $2 \times 8$ & $2 \times 8$ & $2 \times 8$ & $2 \times 8$ & $3 \times 8$ & $3 \times 8$ & $3 \times 10$ & $3 \times 10$ \\
\hline Sprint from a standing position (m) & $5 \times 20$ & $6 \times 20$ & $6 \times 20$ & $6 \times 20$ & $8 \times 20$ & & & \\
\hline \multicolumn{6}{|l|}{ Sprint from a flying start (m) } & $8 \times 10$ & $5 \times 30$ & $5 \times 15$ \\
\hline & \multicolumn{8}{|c|}{ Training session } \\
\hline Exercise & 9 & 10 & 11 & 12 & 13 & 14 & 15 & 16 \\
\hline $\begin{array}{l}2 \text { legged jumps (without bending the } \\
\text { knees) up the stairs } 1 \text { step }\end{array}$ & $4 \times 20$ & $4 \times 20$ & $5 \times 20$ & $5 \times 20$ & & & & \\
\hline $\begin{array}{l}2 \text { legged jumps (while bending the } \\
\text { knees) up the stairs } 2 \text { steps }\end{array}$ & & & & & $4 \times 20$ & $4 \times 20$ & $5 \times 20$ & $5 \times 20$ \\
\hline $\begin{array}{l}2 \text { legged jumps as far as possible (while } \\
\text { bending the knees) }\end{array}$ & $4 \times 10$ & $4 \times 10$ & $4 \times 10$ & $4 \times 10$ & $4 \times 12$ & $4 \times 12$ & $4 \times 12$ & $4 \times 12$ \\
\hline $\begin{array}{l}\text { Hop on one leg, short and quickly up } \\
\text { the stairs } 1 \text { step }\end{array}$ & $3 \times 10$ & $3 \times 10$ & $3 \times 10$ & $3 \times 10$ & & & & \\
\hline $\begin{array}{l}\text { Hop on one leg, short and quickly up } \\
\text { the stairs } 2 \text { steps }\end{array}$ & & & & & $3 \times 10$ & $3 \times 10$ & $3 \times 10$ & $3 \times 10$ \\
\hline Jump shot movement & $3 \times 8$ & $3 \times 8$ & $3 \times 10$ & $3 \times 10$ & $3 \times 10$ & $3 \times 10$ & $3 \times 10$ & $3 \times 10$ \\
\hline Sprint from 5 m sideways start (m) & $6 \times 30$ & $6 \times 15$ & $8 \times 30$ & $8 \times 15$ & & & & \\
\hline Sprint from 3 m backwards start (m) & & & & & $6 \times 30$ & $6 \times 20$ & $8 \times 30$ & $8 \times 20$ \\
\hline
\end{tabular}

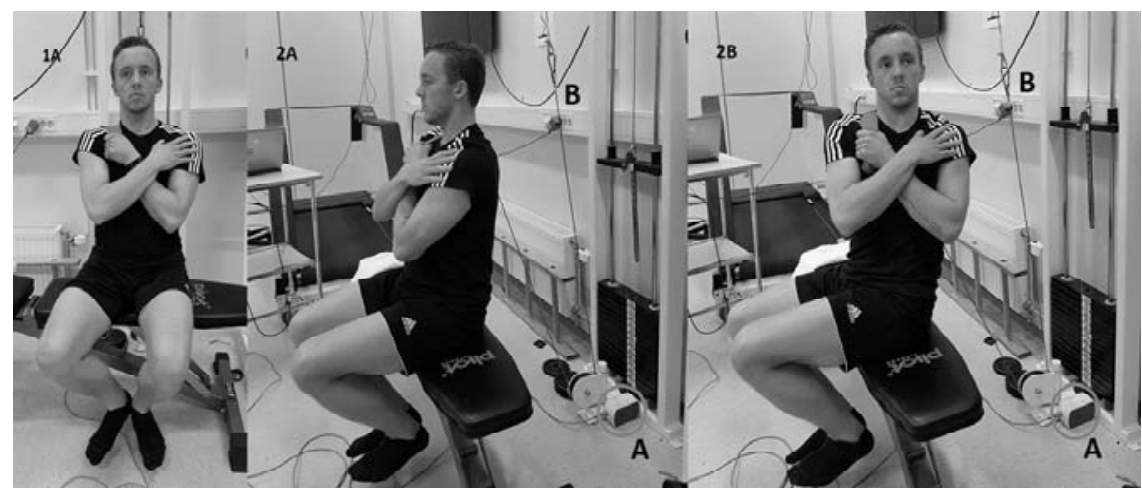

Figure 1

Core strength test. 1A: Frontal view of the starting position. 2A: Side view of the starting position, with a linear encoder $(A)$ and apparatus with addable weights $(B) .2 B$ :

Side view of the ending position. 


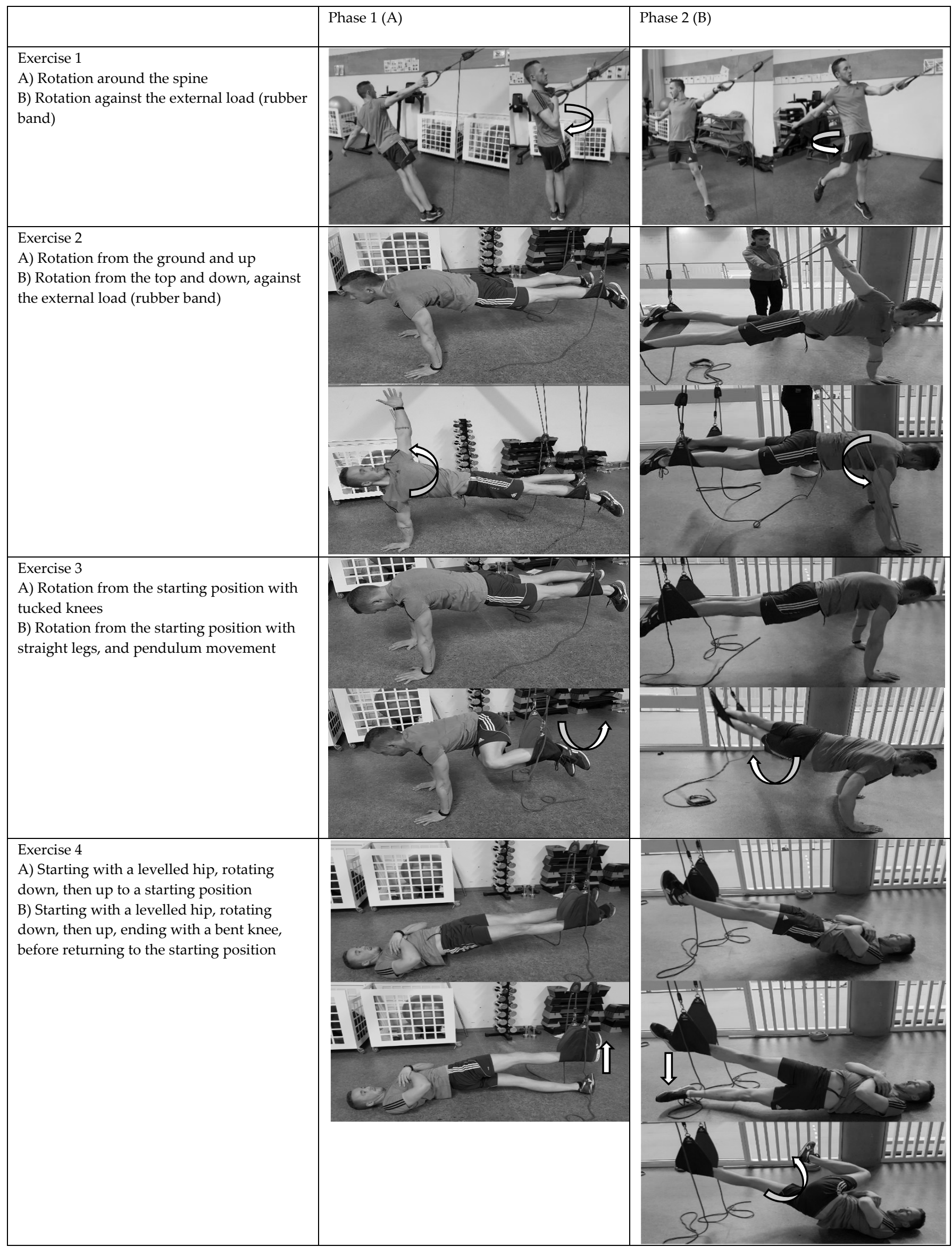

Figure 2

Sling-based training protocol exercises. Person in pictures was not a participant 


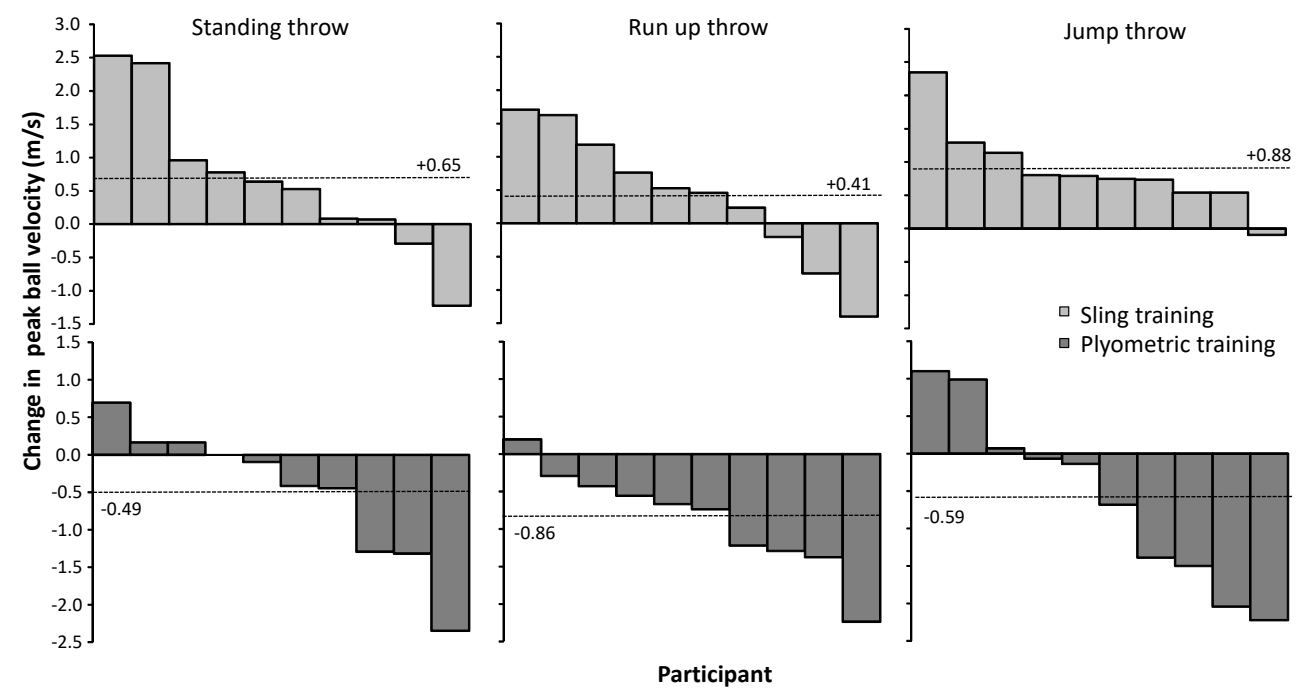

Figure 3

Change in maximal ball shooting velocity from pre- to post-test per subject for $7 \mathrm{~m}$ penalty shots, $7 \mathrm{~m}$ shots with two step run-up, and jump shot (also with a two-step run-up) at $8 \mathrm{~m}$, with the average change per group indicated by a horizontal line.

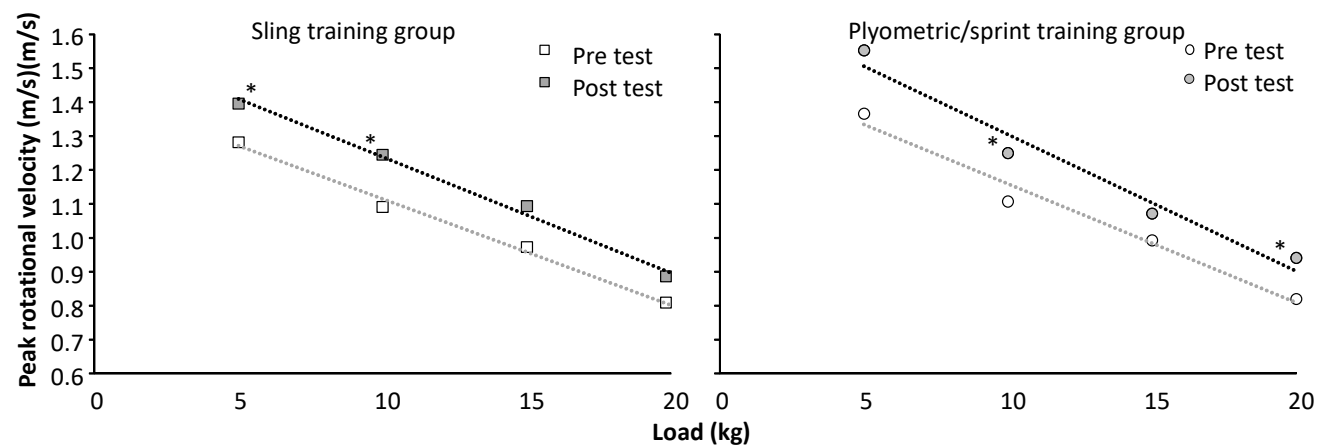

Figure 4

Peak rotational velocity with the four different loads, averaged per group for pre- to post-test. * Indicates a significant increase in velocity with this load from the pre- to post-test at the $p<0.05$ level. 


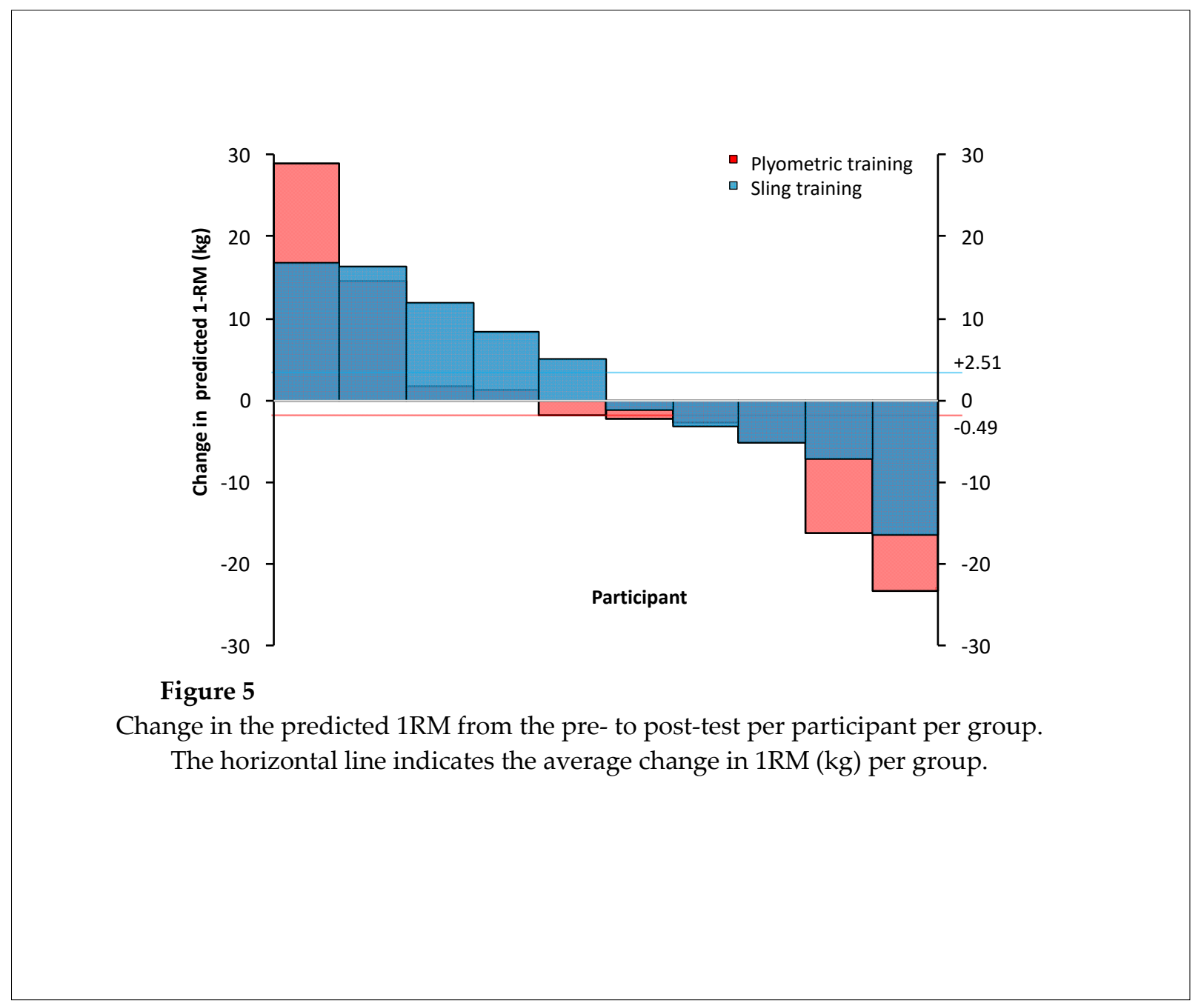

There was no significant effect of time for maximal ball velocity for any of the types of shots $\left(p \geq 0.26, \eta^{2} \leq 0.07\right)$. However, a group effect over time was found for the $7 \mathrm{~m}$ standing shot without a run-up $\left(p=0.024, \eta^{2}=0.25\right)$, the run-up shot $(p=$ $\left.0.004, \eta^{2}=0.38\right)$ and the jump shot $\left(p=0.003, \eta^{2}=\right.$ 0.40). Post hoc comparison revealed that the slingbased training group had significant increases of 3.3, 1.9 and $4.4 \%$ in the maximal ball velocity of the $7 \mathrm{~m}$ standing shot, run-up and jump shot, respectively, while the plyometric/sprint training group had decreases of 2.4, 3.9, and $2.8 \%$ in these shots (Figure 3).

A significant effect of time was found for the maximal rotational velocity with all four loads $\left(p \leq 0.032, \eta^{2} \geq 0.21\right.$, Figure 4), but not for the predicted 1RM ( $p=0.81, \eta^{2}<0.01$, Figure 5). No significant effect was found between groups for the predicted $1 \mathrm{RM}$ (sling-based training: $+6.9 \%$, control group: $-1.3 \%$ ) or any of the maximal rotational velocities $\left(p \geq 0.56, \eta^{2}<0.02\right)$. Post hoc comparisons revealed significant increases in maximal rotational velocity with $10 \mathrm{~kg}$ in both groups, and with $20 \mathrm{~kg}$ in the plyometric/sprint training group. No significant correlations were found between change in maximal ball velocity in any of the types of shots and change in any of the core strength variables $(p \geq 0.13)$.

\section{Discussion}

The purpose of this study was to determine the effect of a core strength program, mainly composed of rotational exercises in slings, on shooting velocity in female handball players. The main findings were that there was a significant group effect over time: sling-based training over 8 weeks during the competitive season increased ball velocity on average by $3.2 \%$, whereas shooting performance decreased by $3 \%$ in the control group. However, both training 
groups increased peak rotational velocity with different loads, but not the calculated 1RM core strength after the training period.

The increase in ball velocity after the intervention period supports the hypothesis that shooting performance can be increased through a core strength program, using rotational movements in slings. The increase varied from $1.9 \%$ for the running shot to $4.4 \%$ for the jump shot, with a positive response in at least $70-90 \%$ of the involved athletes (Figure 3). This is in accordance with previous findings of sling-based training in fast-discrete complex movements (Goulet and Rogowski, 2018; Saeterbakken et al., 2011; Seiler et al., 2006; Stray-Pedersen et al., 2006). However, most of these earlier studies were performed with younger athletes, a lower level of competition (13-16 years old) or in the preparation phase of the competitive season. The present study indicates that sling-based training can increase in-season ball velocity in handball players, whereas during the competitive season, shooting performance normally decreases gradually when no extra upper body strength training is included, besides normal handball training (van den Tillaar et al., 2015). In the present study, this was shown by a decrease of around $3 \%$ in shooting performance of the plyometric/sprint training group (control group).

It was expected that sling-based training should increase core strength. However, it seemed that only peak rotational velocity increased when using different loads during the core strength test, while the calculated 1RM load did not increase significantly after the intervention period. An explanation for this is that, with lower loads, a greater increase in rotational velocity was observed than with heavy loads, which caused a steeped regression line in the sling-based training group. This resulted in the same calculated 1RM (Figure 4). This was especially observed through increases in the peak rotational velocities with the $5(\mathrm{p}=0.09)$ and $10 \mathrm{~kg}$ loads in the sling-based training group. Thus, it seems that sling-based training had the mostly positive effect at low loads in the load-velocity relationship, while plyometric/sprint training could also increase core strength at higher loads. Furthermore, there was no difference in the increase in peak rotational velocity between the sling-based training group and the plyometric/sprint group. This could be due to the fact that throughout plyometric training, the core is also trained to keep balance during the different jumps (Hill and Leiszler, 2011). The core has to be active during these jumps to transfer the forces from the lower extremities to the upper body, and to keep the forces between the two parts of the body in balance to maintain stability, as Kibler et al. (2006) suggest.

There was no relationship between the change in maximal core strength and the change in ball velocity after the training period, which may indicate that maximal core strength of the upper torso is not the factor that increases ball velocity. However, the core strength test used is perhaps not the best test to detect small changes in maximal core strength for shooting since participants were tested while sitting, which results in a fixed pelvis. Thereby, only rotational velocities of the upper torso were possible, which constrains the possibility to detect the impact of sling-based training upon maximal rotational movement of the pelvis, timing variables between core segments, and possible power transfer from the pelvis and the upper torso. However, unpublished electromyography data on one subject on the shooting techniques, core strength tests and sling-based exercises, that were collected after the completion of the intervention, showed that three sling-based exercises led to the muscle activation of the external oblique that was close to double that of core-strength exercise and was on average about three times the maximal activation during shooting. This may suggest that muscle activation is high enough to increase maximal core strength over time and enhance core contribution during shooting. Probably, the amount of sling-based training in the present study was not sufficient (only 320 minutes over eight weeks), as it was only half of the training time compared with earlier sling-based training studies (Saeterbakken et al., 2011; Seiler et al., 2006), to enhance core strength to a greater extent, compared with the control group.

Earlier studies have found that pelvic and trunk rotation are important contributors to achieve maximal ball velocity in handball players (Wagner et al., 2010; Wagner et al., 2011; Wagner et al., 2012b) through the transfer of energy from the pelvis via the trunk to the upper extremity (Jöris et al., 1985). While some studies (Fradet et 
al., 2004; van den Tillaar and Ettema, 2007, 2009b) have also shown that joint movements like internal shoulder rotation and elbow extension are important contributors to ball velocity, Wagner et al. (2011) found that these movements were close to identical between shooting techniques. Due to the differences in maximal ball velocity in the techniques tested, they argued that other variables (pelvic and trunk rotation) prior to movement in the upper extremity impacted the chain of movement and ball velocity. Therefore, it may be important to include tests on how training impacts timing between different joint movements (Wagner et al., 2012a), possible increases in range of motion (van den Tillaar and Ettema, 2009a), and power transfer between segments. Through further kinematical investigation on how such training may interfere with the execution of a fast-discrete complex movement, it will become clearer how training alters technical execution of such movements.

The current study is not without limitations. The focus of sling-based training was to stimulate the pelvic and trunk rotation strength necessary for shooting. However, a complete shooting sequence involves both the external and internal rotation of both the hips and trunk, and tilting the core in various directions, together with various arm joint movements (van den Tillaar and Ettema, 2009b; Wagner et al., 2011). To work on these core movements, exercises were selected based on their rotational characteristics around the longitudinal axis (Figure 2), and the timing of various muscle involvement, thereby altering the proximal-to-distal sequence positively. These possible altered kinematics could explain enhanced shooting performance after sling-based training. However, no kinematical analysis was performed from pre- to post test that could confirm if altered kinematics occurred. To the best of our knowledge, not many studies have investigated the effects of strength training upon changes in kinematics in handball shooting. Only van den Tillaar and Marques (2011) investigated this issue using a pulley device and different weighed balls, and found that internal shoulder rotation velocity was one of the few joint movements that related to changes in maximal ball velocity. Therefore, in the future, threedimensional kinematic studies, together with electromyography measurements of the involved muscles should be conducted to investigate how sling-based training influences maximal ball velocity.

In conclusion, sling-based training with rotational core exercises that progressively increase in difficulty and resistance can improve maximal ball velocity in female handball players during the competitive season. However, this increased ball velocity is probably not caused by increased maximal core strength in the upper torso. Further studies should be conducted to investigate how such exercises may impact strength in the pelvic area, and if possible, the degree of power transfer from the pelvis to the trunk in such cases. Also, the timing between joint movements involving the core is probably responsible for the enhancement in ball velocity.

\section{Conclusion}

Based on the findings of the present study, we may conclude that sling-based training with rotational exercises can improve shooting performance in outfield handball players in the competitive season, yet these increases are not a result of increased maximal core strength. Strength coaches can incorporate sling-based exercises, exposing the joints to destabilization forces during training. This may encourage an effective neuromuscular pattern, increase force production and improve a highly specific performance task such as shooting. Furthermore, the kinetics of shooting are similar to other sports involving segmental summation. It is therefore likely that the improvements observed in the present study may translate to other sports and improve specific performance tasks.

\section{References}

Cohen J. Statistical power analysis for the behavioral sciences. Hillsdale, NJ, England: Lawrence Erlbaum Associates; 1988

Fradet L, Botcazou M, Durocher C, Cretual A, Multon F, Prioux J, Delamarche P. Do handball throws always exhibit a proximal-to-distal segmental sequence? J Sports Sci, 2004; 22: 439-447 
Genevois C, Berthier P, Guidou V, Muller F, Thiebault B, Rogowski I. Effects of 6-week sling-based training of the external-rotator muscles on the shoulder profile in elite female high school handball players. $J$ Sports Rehab, 2014; 23: 286-295

Goulet C, Rogowski I. Sling-based exercise for external rotator muscles: effects on shoulder profile in young recreational tennis players. J Sport Rehab, 2018; 27: 30-36

Hill J, Leiszler M. Review and Role of Plyometrics and Core Rehabilitation in Competitive Sport. Curr Sports Med Reports, 2011; 10: 345-351

Jöris HJJ, Edwards van Muyen AJ, van Ingen Schenau GJ, Kemper HCG. Force velocity and energy flow during the overarm throw in female handball players. J Biomech, 1985; 18: 409-414

Kibler WB, Press J, Sciascia A. The role of core stability in athletic function. Sports Med, 2006; 36: 189-198

Manchado C, Garcia-Ruiz J, Cortell-Tormo JM, Tortosa-Martinez J. Effect of core training on male handball players' throwing velocity. J Hum Kinet, 2017; 56: 177-185

Marques MC, Pereira A, Reis IG, van den Tillaar R. Does an in-Season 6-Week Combined Sprint and Jump Training Program Improve Strength-Speed Abilities and Kicking Performance in Young Soccer Players? J Hum Kinet, 2013; 39: 157-166

Saeterbakken AH, van den Tillaar R, Seiler S. Effect of core stability training on throwing velocity in female handball players. J Strength Cond Res, 2011; 25: 712-718

Scibek JS. The effect of core stabilization training on function performance in swimming. Microform Publications, Eugene, University of Oregon; 1999

Seiler S, Skaanes PT, Kirkesola G, Katch FI. Effects of sling exercise training on maximal clubhead velocity in junior golfers. Med Sci Sports Exerc, 2006; 38: S286

Stanton R, Reaburn PR, Humphries B. The effect of short-term Swiss ball training on core stability and running economy. J Strength Cond Res, 2004; 18: 522-528

Stray-Pedersen JI, Magnussen R, Kuffel E, Seiler S, Katch F. Sling exercise training improves balance, kicking velocity and torso stabilization strength in elite soccer players. Med Sci Sports Exerc, 2006; 38: S243

van den Tillaar R, Ettema G. Instructions emphasizing velocity, accuracy, or both in performance and kinematics of overarm throwing by experienced team handball players. Perc Mot Skills, 2003; 97: 731742

van den Tillaar R, Ettema G. A three-dimensional analysis of overarm throwing in experienced handball players. J Appl Biomech, 2007; 23: 12-19

van den Tillaar R, Ettema G. A comparison of overarm throwing with the dominant and nondominant arm in experienced team handball players. Perc Mot Skills, 2009a; 109: 315-326

van den Tillaar R, Ettema G. Is there a proximal-to-distal sequence in overarm throwing in team handball? J Sports Sci, 2009b; 27: 949-955

van den Tillaar R, Marques M. Effect of training on ball release velocity and kinematics in overarm throwing among experienced female handball players. Kin Slov, 2011; 17: 38-46

van den Tillaar R, Waade L, Roaas T. Comparison of the effects of 6 weeks of a squat training programme upon different performance tests in adolescentteam handball players. Acta Kin Univ Tart, 2015; 21: 7588

Wagner H, Buchecker M, von Duvillard S, Muller E. Kinematic comparison of team handball throwing with different arm positions. Int J Sports Physiol Perf, 2010; 5: 469-483

Wagner H, Pfusterschmidt J, von Duvillard SP, Müller E. Performance and kinematics of various throwing techniques in team-handball. J Sports Sci Med, 2011; 10: 73-80

Wagner H, Pfusterschmied J, von Duvillard S, Muller E. Skill-dependent proximal-to-distal sequence in team-handball throwing. J Sports Sci, 2012a; 30: 21-29 
Wagner H, Pfysterschmiedd J, Klous M, von Duvillard S, Muller E. Movement variability and skill level of various throwing techniques. Hum Mov Sci, 2012b; 31: 78-90

Willardson JM. Core stability training: applications to sports conditioning programs. J Strength Cond Res, 2007; 21: 979-985

\section{Corresponding author:}

\section{Roland van den Tillaar PhD.}

Department of Sports Science and Physical Education Nord University

Odins veg 23

7603 Levanger

Norway

Phone: +47-5767 1883

Fax: 0047-7411 2001

E-mail: roland.v.tillaar@nord.no 